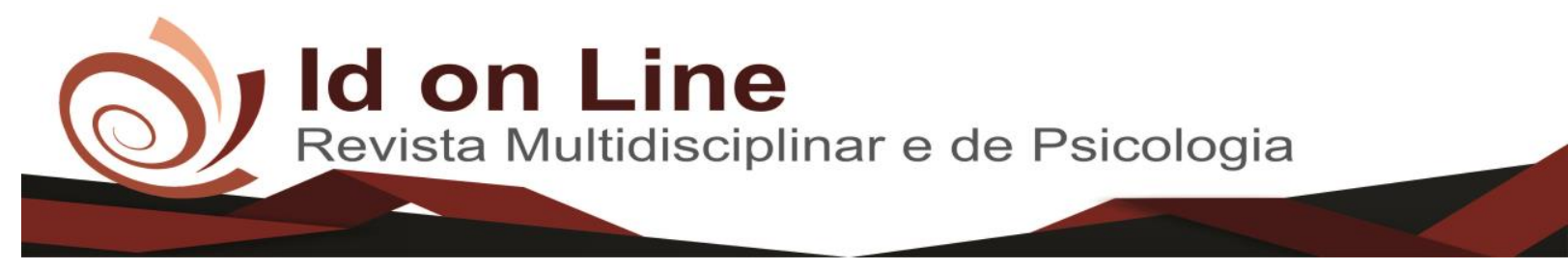

Artigo de Revisão

\title{
Triptofano no Sono: \\ Uma Revisão Sistemática baseada no Método PRISMA
}

Leonardo da Silva Lima'; Carla Pequeno da Silva²;

\begin{abstract}
Resumo: O sono é um processo biológico que apresenta alternância com períodos de vigília, modulado por hormônios e funções neuronais, tais como mecanismos fisiológicos que modificam a temperatura corporal, produção de hormônios e função cardíaca. Anormalidades no sono podem acarretar em má qualidade de vida e a longo prazo em doenças metabólicas e cardiovasculares. Acredita-se que um aliado para o tratamento das desordens do sono seja o triptofano (TRP), que por sua vez, é um aminoácido precursor da melatonina e serotonina. O TRP e alguns dos seus produtos metabólicos, como serotonina e melatonina, participa da regulação do humor, do sono e dos ritmos circadianos e são usados para tratar insônia e apnéia do sono. $\mathrm{O}$ objetivo desta pesquisa foi identificar a eficácia do uso do triptofano no sono através de uma revisão sistemática no modelo PRISMA. A revisão sistemática foi realizada a partir de três bases de dados (PubMed, Lilacs e Scielo). Foram encontrados 1.034 artigos, sendo 876 da PubMed, 157 da Lilacs e 1 da Scielo, após a aplicação dos critérios de elegibilidade foram incluídos 8 artigos na revisão. Os estudos mostraram efeitos positivos da suplementação do triptofano no sono, este aminoácido mostrou-se ser eficaz até mesmo no tratamento de distúrbios relacionados ao sono.
\end{abstract}

Palavras-chave: Sono, distúrbio do sono, tratamento, triptofano.

\section{Tryptophan in Sleep: A Systematic Review based on the PRISMA Method}

\begin{abstract}
Sleep is a biological process that alternates with periods of wakefulness, modulated by hormones and neuronal functions, such as physiological mechanisms that modify body temperature, hormone production and cardiac function. Abnormalities in sleep can lead to poor quality of life and long-term metabolic and cardiovascular diseases. It is believed that an ally for the treatment of sleep disorders is tryptophan (TRP), which in turn is an amino acid precursor of melatonin and serotonin. TRP and some of its metabolic products, such as serotonin and melatonin, play a role in the regulation of mood, sleep and circadian rhythms and are used to treat sleep apnea and insomnia. The objective of this research was to identify the effectiveness of the use of tryptophan in sleep through a systematic review in the PRISMA model. The systematic review was performed from three databases (PubMed, Lilacs and Scielo). A total of 1,034 articles were found, of which 876 were PubMed, 157 were Lilacs and 1 Scielo. After the application of the eligibility criteria, 8 articles were included in the review. Studies have shown positive effects of tryptophan supplementation on sleep, this amino acid has been shown to be effective even in the treatment of sleeprelated disorders.
\end{abstract}

Keywords: Sleep, sleep disorder, treatment, tryptophan.

\footnotetext{
${ }^{1}$ Discente do curso de Fisioterapia da Faculdade Independente do Nordeste - FAINOR, Vitória da Conquista/ BA. Email: leooliima4@gmail.com

${ }^{2}$ Fisioterapeuta, Mestra em Saúde Pública, Docente da Faculdade Maurício de Nassau - UNINASSAU, Vitória da Conquista/ Ba. E-mail: carla_rivka@hotmail.com
} 


\section{Introdução}

O sono é um estado funcional, cíclico e pode ser revertido, essencial à vida. Durante o sono, ocorrem no organismo mudanças no parâmetro biológico e na atividade mental dos seres humanos, que correspondem ao comportamento de dormir, com algumas manifestações comportamentais, imobilidade relativa e aumento do limiar de resposta aos estímulos externos (CARSKADON; DEMENT, 2011; MARTINS; MELLO; TUFIK, 2001; PEQUENO, 2017; ROETHS; TOTH, 2000).

O sono e a vigília são intercalados por meio do ritmo circadiano (RC), podendo sofrer variações de acordo com idade, sexo e peculiaridades de cada indivíduo. O RC é regulado de forma homeostática envolvendo citocinas, fatores neuro humorais e endócrinos (CHOKROVERTY, 2010; PEQUENO, 2017).

Anormalidades no padrão do sono podem causar prejuízo em curto ou em longo prazo à saúde dos afetados. Os sintomas relacionados aos transtornos do sono são comuns tanto em adultos quanto em crianças, podem ocorrer problemas tais como: insônia, sonolência excessiva diurna, incapacidade de dormir no momento desejado e eventos anormais durante o sono (NEVES et al, 2013). Os distúrbios relacionados ao sono são prevalentes na sociedade moderna, porém muitos indivíduos permanecem sem diagnóstico e tratamento (FARIAS, 2009). Estima-se que 50 a 70 milhões de norte americanos sofram de transtornos do sono, dos quais 40 milhões são crônicos. Os efeitos em longo prazo de desordens no sono têm sido associados a gama de consequências deletérias para a saúde, tais como: hipertensão, diabetes, obesidade, depressão, ataque cardíaco e acidente vascular cerebral (FARIAS, 2009).

O tratamento dos distúrbios do sono varia de acordo ao acometimento e pode ser medicamentoso, por higiene do sono, utilização de aparelhos e reposição hormonal (NEVES et al, 2013; NUNES, 2002). Neste contexto, o triptofano vem sendo descrito por profissionais da área como uma ferramenta no tratamento de problemas relacionados ao sono (KALUZNA-CZAPLINSKA et al, 2017). Cientistas tem buscado entender a relação entre sono e dieta, esse interesse é oriundo da discussão de que a via enzimática da síntese de melatonina, o precursor da melanina é a serotonina. Isso, por sua vez, é sintetizado por transformação enzimática do triptofano (KACZOR; SKALSKI, 2016).

Sabe-se que hormônios produzidos no cérebro, como serotonina e melatonina, participam da regulação do sono e de ritmos circadianos em seres humanos. A melatonina 
é um composto biológico ativo que possui a função de regular ritmos diurnos, participa do sistema imunológico, reprodutivo e gastrointestinal. Durante a noite a glândula pineal secreta melatonina e assim, regula ritmos circadianos e o padrão do sono (RICHARD et al., 2009; SZCZEPANIK, 2007).

O triptofano é um aminoácido que o organismo humano não produz, sendo adquirido por meio de dieta. No tratamento de distúrbios do sono os pesquisadores acreditam que o triptofano tem efeitos terapêuticos por meio da melatonina, e um de seus benefícios é não reduzir habilidades cognitivas do pacientes durante o uso (RICHARD et al., 2009). Uma hipótese é que a luz solar acelere a síntese de serotonina, isso foi demonstrado nos estudo de Nakade et al., 2012 e Wada et al., 2013, ambos pesquisadores japoneses sugerem que um pequeno almoço rico em triptofano e exposição regular ao sol matinal pode potencializar a secreção de melatonina durante o período noturno, os autores perceberam que essa associação melhorou a qualidade do sono e o tempo para adormecer dos avaliados.

Portanto, iniciar uma reflexão a partir de uma revisão sistemática, em pesquisas publicadas relacionadas ao tema, é de grande relevância para a obtenção do conhecimento da ação do tripanofano no sono e como este pode interferir no desempenho e na qualidade do sono dos seres humanos.

Para isso, esta revisão se baseou no modelo Preferred Reporting Items for Systematic Reviews and Meta-Analyses (PRISMA), que é composta por uma lista de verificação contendo 27 itens e um diagrama de fluxo de quatro fases. A lista inclui itens considerados de suma importância para a comunicação transparente de uma revisão sistemática (LIBERATI et al., 2009).

Essa ferramenta possibilita que os autores realizem um check list dos itens que compõem os dados antes da submissão dos artigos de revisão sistemática como também auxilia os pesquisadores durante a realização do processo de revisão (PÁDULA et al., 2012).

Dessa forma, o objetivo deste estudo foi identificar a eficácia da reposição do triptofano no tratamento de distúrbios do sono através de uma revisão sistemática, utilizando o método PRISMA. Como forma de compreender quais os benefícios do triptofano no sono; analisar o uso do triptofano e seus efeitos no sono. 


\section{Procedimentos metodológicos}

A definição da pergunta e a estratégia utilizada para compor este estudo baseou-se no modelo Population, Intervention Comparison, Outcome (PICO), que em português significa "População, Intervenção, Comparação, Desfecho". Esse modelo é amplamente utilizado na Prática Baseada em Evidências (PBE) e recomendado para revisões sistemáticas (SANTOS et al., 2007).

\section{Estratégia de busca}

A estratégia utilizada neste estudo compreendeu a efetivação de buscas sistemáticas na literatura, a partir das bases de dados PubMed, Lilacs e Scielo. As buscas foram realizadas por artigos disponíveis até o dia 5 de março de 2018. Não foram utilizados filtros por desenho de estudo, tamanho da amostra ou por data, pois a pesquisa pretendeu realizar uma revisão de todos os estudos publicados até a data final da pesquisa. Durante a revisão sistemática, foram utilizadas palavras incluídas nos recursos dos Descritores em Ciências da Saúde (DeCS) e Termos Livres que não foram encontrados no DeCS nem no Medical Subject Headings (MeSH). Foram inseridas algumas variações, as quais resultaram na seguinte combinação: “tryptophan” OR "tryptophan hydroxylase” OR "tryptophan supplementation” $O R$ “Aromatic-L-Amino-Acid Decarboxylases ” $O R$ "Tryptophan Oxygenase " $O R$ “Tryptophan Synthase ”. Tais combinações e variações dos descritores foram utilizadas como forma de ampliar as possibilidades de encontrar resultados significativos referentes ao tema. Os descritores foram escritos na língua inglesa para ampliar o potencial de busca das publicações.

\section{Critérios de elegibilidade}

Durante a revisão sistemática, foram considerados elegíveis os estudos 1) realizados em seres humanos de ambos os sexos em qualquer faixa etária; 2) que abordam o uso do triptofano; 3) publicados nos idiomas português, inglês ou espanhol; 4) disponíveis através de texto completo. Não foram incluídos teses, revisões de literatura, dissertações e monografias. 


\section{Seleção dos artigos}

Os artigos foram baixados em todas as bases de dados escolhidas através do navegador Mozilla Firefox. Para verificar e identificar duplicidade os artigos foram adicionados no software Mendley Desktop. Os arquivos que continham as referências extraídas das bases de dados foram selecionados pelas autoras desta revisão de forma individual e ao final houve a comparação dos resultados, inicialmente com base na leitura do título, e em seguida foi feita a leitura dos resumos. A partir dos resumos selecionados, os artigos completos foram revisados. Os dados de artigos incluídos foram extraídos de forma independente, utilizando um formulário padrão. Após a extração, os dados foram comparados e discutidos para que fossem aplicados os critérios de elegibilidade predefinidos para a inclusão e a exclusão dos estudos.

\section{Extração dos dados}

Após o cumprimento das etapas anteriores, os revisores realizaram a extração dos seguintes dados: autoria; ano de publicação; características das amostras; efeitos do uso do triptofano no sono e os desfechos de cada estudo.

\section{Resultados}

Ao término das buscas nas bases de dados por meio dos descritores anteriormente mencionados, foram encontrados 1.034 artigos, sendo 876 na PubMed, 157 na Lilacs e 1 no Scielo. Com os critérios de elegibilidade para inclusão e exclusão dos estudos, foram excluídos 936 pelo título e 89 trabalhos pelo resumo. Os motivos frequentemente observados para exclusão nesta etapa foram estudos que avaliavam a deficiência do triptofano no sono, não verificando o efeito do uso do triptofano no sono e em outros estudos foram realizados em modelo animal. Também foram encontrados e excluídos estudos de revisão sistemática, meta-análises, artigos incompletos e resumo não disponível.

Dentre os artigos selecionados para revisão do texto completo um apresentou duplicidade e outro não se enquadrava nos critérios de elegibilidade deste estudo. Após 
seleção e aplicação dos critérios de elegibilidade, foram incluídos 7 artigos nesta revisão sistemática. O processo de seleção e aplicação dos critérios de elegibilidade pode ser observado por meio do fluxograma do modelo Prisma na Figura 1.

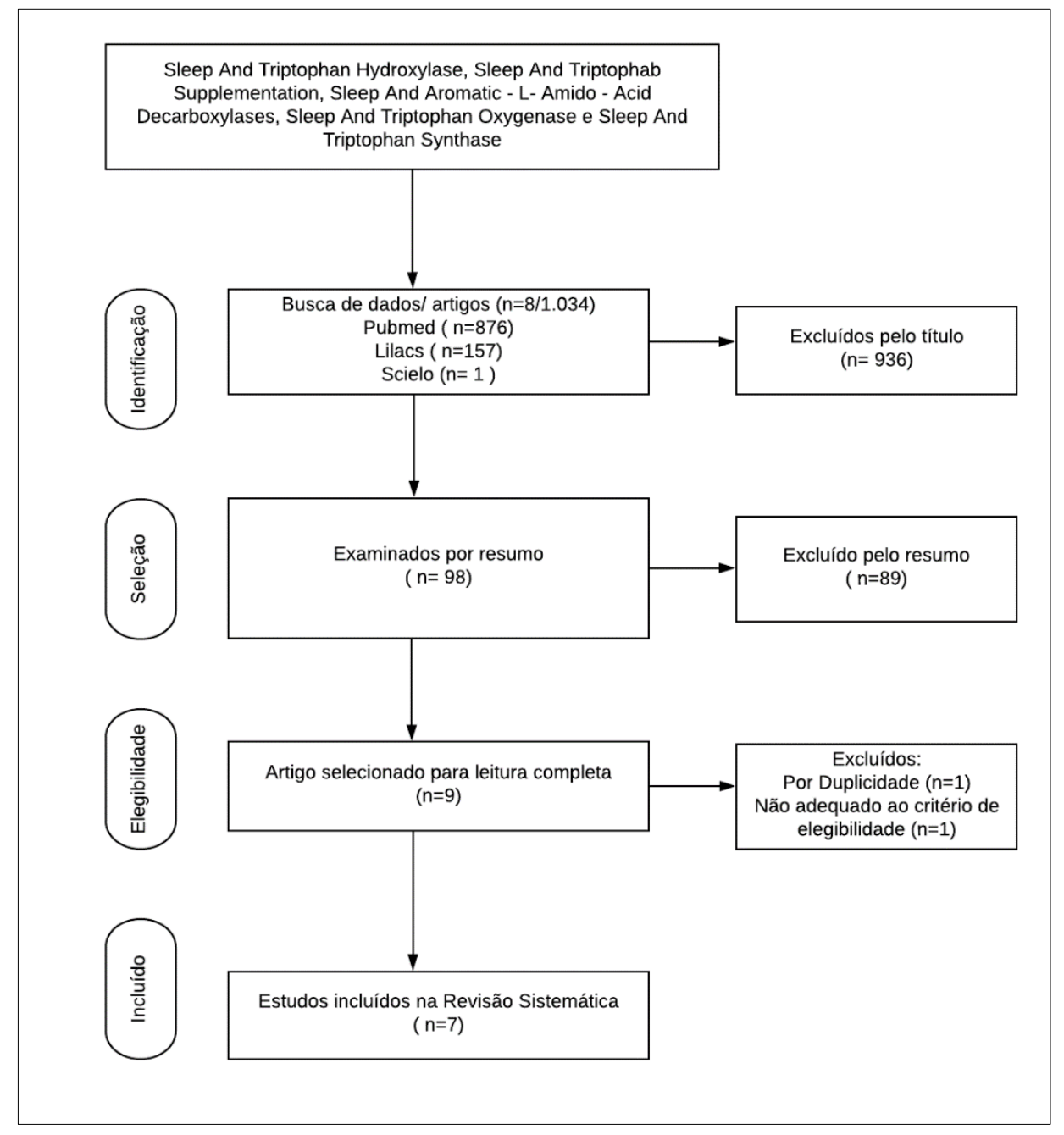

Figura 1. Fluxograma baseado no modelo PRISMA com os resultados da seleção dos artigos.

A Tabela 1 apresenta as características relevantes dos estudos selecionados. Dos sete estudos incluídos, apenas um é randomizado. A maioria dos estudos é transversal. Somente um dos estudos selecionados foi realizado com crianças. O tamanho da amostra variou de forma significativa, de 34 no estudo de Bravo et al (2013) a 1727 na pesquisa de Harada et al (2007). A maioria dos estudos fez uso de questionário para avaliar o uso do triptofano e o sono dos indivíduos estudados, um estudo fez uso de actígrafo para analisar ritmo do sono (BRAVO et al/ 2013). 
Tabela 1 - Principais características dos estudos selecionados

\begin{tabular}{|c|c|c|c|c|c|}
\hline Autor/ano & $\begin{array}{c}\text { População do } \\
\text { estudo }\end{array}$ & Idade & $\begin{array}{l}\text { Desenho do } \\
\text { estudo/ Data } \\
\text { da Coleta }\end{array}$ & $\begin{array}{l}\text { Método do } \\
\text { estudo }\end{array}$ & $\begin{array}{c}\text { Efeito encontrados } \\
\text { do triptofano no } \\
\text { sono }\end{array}$ \\
\hline $\begin{array}{c}\text { Harada et al/ } \\
2007\end{array}$ & $\begin{array}{c}1727 \text { crianças. } \\
\text { Os responsáveis } \\
\text { responderam questionário } \\
\text { para crianças com idade } \\
\text { de } 7 \text { meses a } 8 \text { anos ( } 740 \\
\text { lactantes e } 195 \text { alunos da } \\
\text { primeira e segunda série), } \\
\text { destes } 93 \% \text { foram } \\
\text { respondidos por mães }\end{array}$ & $\begin{array}{l}\geq 7 \text { meses }-15 \\
\text { anos }\end{array}$ & $\begin{array}{l}\text { Transversal/ } \\
\text { junho a } \\
\text { novembro de } \\
2004\end{array}$ & Questionário & $\begin{array}{l}\text { - triptofano absorvido no } \\
\text { café da manhã auxilia as } \\
\text { crianças a manterem o } \\
\text { ritmo diurno e melhora a } \\
\text { qualidade do sono, os } \\
\text { autores supõem que seja } \\
\text { por meio do } \\
\text { metabolismo do triptofano } \\
\text { à serotonina durante o dia e } \\
\text { melatonina à noite }\end{array}$ \\
\hline Wada et al/ 2013 & $\begin{array}{c}94 \text { sujeito de pesquisa, } \\
\text { sexo masculino que } \\
\text { faziam parte de um grupo } \\
\text { de futebol. }\end{array}$ & 19-22 anos & $\begin{array}{l}\text { Coorte/ outubro } \\
\text { a novembro } 2010\end{array}$ & $\begin{array}{l}\text { - Questionário } \\
\text {-Uso de } \\
\text { triptofano }\end{array}$ & $\begin{array}{c}\text { - Uso de triptofano pela } \\
\text { manhã reforçado com } \\
\text { exposição à luz solar } \\
\text { mostrou estimular a síntese } \\
\text { de serotonina } \\
\text { e síntese de melatonina } \\
\text { durante a noite. } \\
\text { - Percebeu-se que } \\
\text { estudantes que fizeram uso } \\
\text { do triptofano durante a } \\
\text { parte da manhã tiveram } \\
\text { melhor qualidade do sono }\end{array}$ \\
\hline Bravo et al/ 2013 & $\begin{array}{l}\text { O estudo foi realizado em } \\
35 \text { voluntários idosos } \\
\text { (26 mulheres e } 9 \text { do sexo } \\
\text { masculino) com } \\
\text { peso normal que sofria de } \\
\text { dificuldades no sono }\end{array}$ & 55- 75 anos & $\begin{array}{l}\text { Coorte/3 } \\
\text { semanas }\end{array}$ & $\begin{array}{l}\text { - Uso de } \\
\text { triptofano } \\
\text { - Actigrafo } \\
\text { - Amostra de } \\
\text { urina }\end{array}$ & $\begin{array}{l}\text { - O consumo de cereais } \\
\text { enriquecidos com } \\
\text { triptofano pode ser } \\
\text { considerado como uma } \\
\text { ferramenta facilitadora para } \\
\text { melhorar o sono dos } \\
\text { idosos }\end{array}$ \\
\hline $\begin{array}{l}\text { Fukushige et al/ } \\
2014\end{array}$ & $\begin{array}{l}40 \text { homens universitários } \\
\text { participaram do estudo }\end{array}$ & 20-22 anos & $\begin{array}{l}\text { Coorte/17 até } 21 \\
\text { de setembro, } \\
2012 \text { (com } \\
\text { duração de } 5 \text { dias } \\
\text { e } 4 \text { noites) }\end{array}$ & $\begin{array}{l}\quad \text { - Uso de } \\
\quad \text { triptofano } \\
\text { - Questionário } \\
\text { - Coleta de } \\
\text { melatonina pela } \\
\text { saliva }\end{array}$ & $\begin{array}{c}\text { - Na noite, a secreção de } \\
\text { melatonina mudou } \\
\text { significativamente } \\
\text { e indicou que um pequeno- } \\
\text { almoço rico em triptófano } \\
\text { e exposição à luz durante o } \\
\text { dia tem o potencial de } \\
\text { aumentar a sintese de } \\
\text { melatonina }\end{array}$ \\
\hline Wang et al, 2016 & $\begin{array}{l}80 \text { voluntários } \\
\text { participaram } \\
\text { do estudo }\end{array}$ & $\begin{array}{l}>30 \\
\text { anos }\end{array}$ & $\begin{array}{l}\text { Randomizado } \\
\text { duplo cego } \\
\text { controlado por } \\
\text { placebo/ }\end{array}$ & $\begin{array}{l}\text { - Questionário } \\
\text { - Uso de } \\
\text { triptofano }\end{array}$ & $\begin{array}{l}\text { - O triptofano mostrou-se } \\
\text { eficaz no tratamento de } \\
\text { distúrbios do sono em } \\
\text { indivíduos em tratamento } \\
\text { de dependência química }\end{array}$ \\
\hline $\begin{array}{l}\text { Lieberman et al, } \\
2016\end{array}$ & $\begin{array}{c}\text { Participaram do estudo } \\
29.687 \text { adultos ( } 15.031 \\
\text { homens e } 14.656 \\
\text { mulheres) }\end{array}$ & $>19$ anos & $\begin{array}{c}\text { Transversal } \\
\text { Coleta por meio } \\
\text { de dados } \\
\text { secundários } \\
\text { /2001 a } 2012\end{array}$ & $\begin{array}{c}\text { - Uso de } \\
\text { triptofano } \\
\text { - Questionário }\end{array}$ & $\begin{array}{l}\text { - Relacionado com maior } \\
\text { duração do sono }\end{array}$ \\
\hline $\begin{array}{c}\text { Nagashima et al, } \\
2017\end{array}$ & $\begin{array}{c}12 \text { voluntários } \\
\text { participaram do estudo }\end{array}$ & $>20$ anos & Transversal & $\begin{array}{c}\text { - Questionário } \\
\text { - Uso de } \\
\text { triptofano } \\
\text { - Coleta de urina }\end{array}$ & $\begin{array}{c}\text { - Em um dia de } \\
\text { suplementação de tripofano } \\
\text { os autores não observaram } \\
\text { efeito significativo sobre a } \\
\text { secreção noturna de } \\
\text { melatonina. No entanto, a } \\
\text { exposição solar diurna } \\
\text { aumentou os níveis de } \\
\text { melatonina }\end{array}$ \\
\hline
\end{tabular}

Fonte: Pesquisa dos autores, 2018 


\section{Discussão}

A presente revisão sistemática mostrou que existe uma relação positiva entre o uso de triptofano e sono, principalmente quando usado logo no período da manhã e com posterior exposição à luz solar. Foram encontrados poucos estudos e alguns apresentaram limitações metodológicas.

Observou-se heterogeneidade nos estudos principalmente na forma de avaliar o uso de triptofano e o sono, apesar de a maioria dos autores terem aplicado questionário e alguns avaliarem os marcadores bioquímicos a composição das amostras e o método aplicado se diferem entre os estudos.

O estudo de Harada et al (2007) foi o único que aplicou apenas questionário e foi realizado com lactantes, crianças e adolescentes. Apesar de ser constituído com uma amostra numerosa, um víeis pode ter ocorrido, já que o sono dos lactantes, das crianças e dos adolescentes é diferente, pois suas necessidades fisiológicas são distintas, bem como o período de latência do sono, sono REM, eficiência do sono, estádios do sono (BERRY et al, 2012; TUFIK , 2008). Sendo assim, a forma de avaliação do sono deve ir de acordo à peculiaridade da população estudada. Outro fator que pode gerar víeis na pesquisa supracitada é a alimentação, o lactante geralmente alimenta-se de forma diferente quando comparado com crianças e adolescentes, então a quantidade do triptofano pode ser menor ou maior ao depender da quantidade consumida e do alimento ingerido, uma forma para evitar esse víeis teria sido a mensuração em gramas do triptofano consumido por cada voluntário do estudo, isso só seria possível se a pesquisa controlasse a quantidade de triptofano ingerida.

A maioria dos estudos incluídos nessa revisão sistemática obtiveram informações sobre o sono por meio de questionário, recurso frequentemente utilizado em pesquisa do sono, por ser um método de baixo custo e fácil aplicação. Todavia, a validade das informações de questionário deve ser criteriosa, usar instrumentos validados e aplicar de maneira adequada. Os indivíduos avaliados de forma subjetiva podem apenas relatar o que acreditam ser socialmente aceitável, facilitando o risco de víeis.

Somente um estudo (Bravo et al/2013), fez uso de uma avaliação objetiva do sono, por meio da actigrafia, que por sua vez, é um exame que utiliza um dispositivo o actígrafo para registrar os movimentos dos indivíduos avaliados durante um período de 24 horas, permitindo 
analisar os padrões do sono e ritmo circadianos (MORGENTALHER et al, 2007). O exame de actigrafia é útil para o estudo em pacientes que apresentam dificuldade para dormir em laboratório do sono, como criança e idoso. Todavia, a actigrafia tem como limitação a necessidade do uso contínuo do aparelho durante os dias de avaliação, sendo que no período do exame o actígrafo é retirado apenas no momento do banho (TOGEIRO, SMITH, 2005). Na pesquisa de Bravo et al ( 2013) os voluntários fizeram uso do aparelho por três semanas.

Para avaliar suplementação do triptofano os estudos observaram ou incrementaram este aminoácido na dieta dos voluntários. Apenas um estudo foi randomizado, duplo-cego e controlado por placebo e comprovou que o triptofano pode ser eficaz no tratamento de distúrbios relacionados ao sono (Wang et al, 2016).

O estudo de Lieberman et al, (2016) observou além da relação entre triptofano e sono, a segurança do uso deste aminoácido na dieta dos indivíduos e ao final sugere que doses elevadas podem melhorar a duração do tempo de sono.

Os resultados aqui encontrados, permitem inferir que, o uso do triptofano é benéfico à saúde dos indivíduos e é um importante aliado do sono, melhorando sua duração e qualidade.

\section{Considerações finais}

Os estudos mostraram efeitos positivos da suplementação do triptofano no sono, este aminoácido mostrou-se ser eficaz até mesmo no tratamento de distúrbios relacionados ao sono.

\section{Referências}

BERRY, R. B. et al. Rules for scoring respiratory events in sleep: update of the 2007 aasm manual for the scoring of sleep and associated events. J Clin Sleep Med, v. 8, n. 5, p. 597-619, 2012.

CARSKADON, M.A; DEMENT, W.C. Monitoring and staging human sleep. Elsevier Saunders. 2011.

CHOKROVERTY, S. Overview of sleep \& sleep disorders. Indian J Med Res. 2010.

FARIAS, C. A. et al. Autonomic modulation in patients with congenital generalized lipodystrophy (Berardinelli-Seip syndrome). Europace, v. 11, n. 6, p. 763-9, Jun 2009. 
KACZOR, M., AND M. SKALSKI. Treatment of behavioral sleep problems in children and adolescents - literature review. Psychiatr Pol. V.50, p.517-84. 2016.

KAŁUŻNA-CZAPLIŃSKA, J et al. How important is tryptophan in human health?. Critical reviews in food science and nutrition, p. 1-17, 2017.

LIBERATI, A. et al. The PRISMA statement for reporting systematic reviews and metaanalyses of studies that evaluate health care interventions: explanation and elaboration. Annals of Internal Medicine, Philadelphia, v. 151, n. 4, p. W65-W94, 2009.

MARTINS, P.J.F; MELLO,M.T.; TUFIK,S . Exercício e sono. Rev Bras Med Esporte, São Paulo, v. 7, n. 1, p. 28-36. Jan/Fev. 2001.

MORGENTALHER, T.I, et al. Practice parameters for the use of actigraphy in the assessment of sleep and sleep disorders. Sleep. v.30, n.4, p.519-27. 2007.

NAKADE, M.,et al. Can breakfast tryptophan and vitamin B6 intake and morning exposure to sunlight promote morning-typology in young children aged 2 to 6 years? J Physiol Anthropol. V.31, n. 11. 2012

NEVES, G.S.M. L. et al. Transtornos do sono: visão geral. Rev Bras Neurol. v.49, n.2, p. 57 $71,2013$.

NUNES, M. L. Distúrbios do sono. Jornal de Pediatria, v. 78, n. 1, p. 63-72, 2002.

PÁDULA, R. S. et al. Análise da apresentação textual de revisões sistemáticas em fisioterapia publicadas no idioma português. Revista Brasileira de Fisioterapia, São Carlos, v. 16, n. 4, p. 281-288, 2012.

PEQUENO, C.S. Avaliação do sono em indivíduos com lipodistrofia generalizada congênita. f.103. Dissertação (Mestrado em Saúde Pública) - Universidade Federal do Ceará, Fortaleza. 2017.

RICHARD, D. M., M. et al. L-Tryptophan: Basic metabolic functions, behavioral research and therapeutic indications. Int J Tryptophan Res.v. 2, p.45-60. 2009.

ROETHS, T; ROTH, T. Sleep-wake state and memory function. Sleep. v.23, n.3, p.64- 68. Mai .2000 .

SANTOS, C. M. C.; PIMENTA, C. B. A.; NOBRE, M. R. C. A estratégia PICO para a construção da pergunta de pesquisa e busca de evidências. Revista Latino-Americana de Enfermagem, Ribeirão Preto, v. 15, n. 3, p. 508-511, 2007.

SZCZEPANIK, M.Melatonin and its influence on immune system. J Physiol Pharmacol. V. 58S6, p. 115-24. 2007. 
TOGEIRO, S. M. G.P; SMITH. A.K. Métodos diagnósticos nos distúrbios do sono. Rev Bras Psiquiatr. v.27.p.8-15.2005.

TUFIK, S. Medicina e biologia do sono. Editora Manole Ltda, 2008.

WADA, K. S.et al. A tryptophan-rich breakfast and exposure to light with low color temperature at night improve sleep and salivar melatonin level in Japanese students. J Circadian Rhythms. V.11, n.4.2013.

\section{Como citar este artigo (Formato ABNT):}

LIMA, Leonardo da Silva; SILVA, Carla Pequeno da. Triptofano no sono: uma revisão sistemática baseada no método PRISMA. Id on Line Rev.Mult. Psic., 2018, vol.12, n.42, Supl. 1, p. 397-407. ISSN: 1981-1179.

Recebido: 06/11/2018;

Aceito: 08/11/2018 